\title{
Do female Norway rats form social bonds?
}

Schweinfurth, M.K. ${ }^{1 *}$; Neuenschwander, J.; Engqvist, L. ${ }^{1}$; Schneeberger, K. ${ }^{1}$; Rentsch, A.K. ${ }^{1}$; Gygax, M. ${ }^{1}$; \&

Taborsky, M. ${ }^{1}$

${ }^{1}$ Behavioural Ecology, Institute of Ecology and Evolution, University of Bern, Wohlenstrasse 50a, CH-3032 Hinterkappelen, Switzerland

* Correspondence: manon.schweinfurth@iee.unibe.ch / phone: +41316319151

Social bonds reflect specific and enduring relationships among conspecifics. In some group living animals they have been found to generate immediate and long-term fitness benefits. It is currently unclear how important and how widespread social bonds are in animals other than primates. It has been hypothesized that social bonds may help establishing stable levels of reciprocal cooperation. Norway rats (Rattus norvegicus) reciprocate received help to an unrelated social partner. It is hitherto unknown, however, whether this cooperative behaviour is based on the establishment of social bonds among involved individuals. Norway rats live in social groups that can be very large, hence without bonds it may be difficult to keep track of other individuals and their previous behaviour, which is a precondition for generating evolutionarily stable levels of cooperation based on direct reciprocity. Here we tested whether wild-type female rats form bonds among each other, which are stable both over time and across different contexts. In addition, we scrutinized the potential influence of social rank on the establishment of bonds. Despite the fact that the hierarchy structure within groups remained stable over the study period, no stable social bonds were formed between group members. Apparently, social information from consecutive encounters with the same social partner is not accumulated. The lack of long-term social bonds might explain why rats base their decisions to cooperate primarily on the last encounter with a social partner, which may differ from other animals where cooperation is based on the existence of long-term social bonds.

Keywords: Norway rats, social bonds, hierarchy, peer relationship 


\section{Significance Statement}

Social bonds have been hypothesized to favour reciprocal cooperation. Norway rats reciprocate help received from a social partner, but it is hitherto unclear whether they form social bonds that might further such cooperative behaviour. Here we tested whether female Norway rats engage in social relationships with a same-sex partner, which are stable over time and across contexts. In contrast to the hypothesized existence of bonds among long-term group members, our results provide no evidence that rats form specific social relationships. Rather than accumulating social information into social bonds, rats apparently base their decision to cooperate merely on the outcome of recent encounters.

\section{Introduction}

Group-living is widespread in animals. It may enhance foraging success (Beauchamp 1998), safety from predators (Foster and Treherne 1981), thermoregulation (Gilbert et al. 2006), energy economy (Herskin and Steffensen 1998), mating opportunities (Wagner 1992), and offspring care (Koenig and Dickinson 2016). In turn, gregarious animals may suffer from increased competition for resources (food: Janson 1988; mates: Wedell et al. 2002), disease transmission (Côté and Poulinb 1994), and the risk of infanticide (Crockett and Janson 2000). To optimize the benefits of group living, individuals can engage in repeated beneficial interactions with a subset of group members (Melis et al. 2006a; Schino and Aureli 2016). Such "social bonds" between individuals may reflect an adaptive strategy increasing the benefits of group living while limiting its costs (Silk et al. 2010).

Social bonds have been referred to as close associations, long-term alliances, affiliative relationships, or friendships (see Silk 2002 for review). They can be defined by the quality and patterning of interactions between group members (Hinde 1976), reflecting dyads that specifically engage with each 
other in a socio-positive way (Massen et al. 2010), and they may be characterized by strong, supportive, equitable, and enduring relationships between two individuals (Silk et al. 2010). Social bonds encompass a variety of relationships that are not limited to kin (e.g., parent-offspring) or mates (pair bonds). Measurements of bond strength commonly take into account the content, quality, frequency, and patterning of interactions, with allogrooming/allopreening and proximity reflecting the most common indices (Silk 2002). There may be short-term and long-term fitness benefits for involved partners; potential short-term benefits include positive outcomes of cooperation (Melis et al. 2006b), reduced harassment (Cameron et al. 2009), and relaxation (Crockford et al. 2013), whereas potential long-term benefits encompass enhanced birth rates (Cameron et al. 2009), increased offspring and adult survival (Silk et al. 2009, 2010), and reduced levels of infanticide (Weingrill 2000).

Social bonds may be expected to emerge predominantly under a combination of several parameters. These include certain sizes and structures of groups, frequent interactions among group members, the capacity of individual recognition and long-term memory, and a potential of receiving marked benefits from bonds. The latter may accrue, for instance, from the reciprocal exchange of services, which may include costly help. In addition, kin discrimination and the existence of a specific rank order have been proposed to be important (Seyfarth and Cheney 2012). Indeed, in some species the strongest bonds take place between kin (for example in elephants: Archie et al. 2006). Furthermore, bonds with high ranking individuals might be more valuable for a subject than bonds with low ranking social partners, as high ranking individuals might variously support and benefit their bonded partner, which might favour bond formation and persistence (see Schino 2001 for a meta-analysis on primates).

Most empirical evidence for close bonds thus far comes from primates (Seyfarth and Cheney 2012), although there is a growing body of literature on other animals (for example in cows (Val-Laillet et al. 2009), dolphins (Lusseau et al. 2003), elephants (Archie et al. 2001), hyenas (Holekamp et al. 1997), horses (Cameron et al. 2009), ravens (Braun and Bugnyar 2012), and voles (Beery et al. 2009)). 
However, in order to understand patterns and functions underlying social bonds, we need information from a greater variety of animals.

Here, we used undomesticated wild-type Norway rats (Rattus norvegicus) as model organism. Rats live in burrow systems (Telle 1966) and are highly social, which is reflected by the fact that isolated rats show depression-like behaviours (Hurst et al. 1997, 1998). Under natural conditions rats live in complex colonies containing up to 200 individuals of different ages, sex, rank, and relatedness (Davis, 1953). Rats individually recognise each other (Gheusi et al. 1997) and form hierarchies among group members (Blanchard et al. 1984). They cooperate with each other, for instance by food sharing, allogrooming, and huddling (Barnett 1963; Krafft et al. 1994), they show mutual coordination (Schuster and Perelberg 2004; Tan and Hackenberg 2016), and they reciprocate food donations and allogrooming with partners (Rutte and Taborsky 2008; Schweinfurth et al. accepted). Reciprocal interactions have been shown to have long-term benefits: rats showing a balanced amount of initiated and received affiliative behaviours live longer and suffer fewer mammary tumours compared to those that show an unequal score (Yee et al. 2008). Thus, rats are apparently good candidates to search for the existence of long-lasting social bonds. Thus far, it remains unclear whether reciprocal exchanges are predominantly shown between closely bonded partners, or whether individuals immediately return received favours without establishing social bonds. We therefore tested whether rats have preferred interaction partners and whether such potential association patterns within a group are stable over time and different contexts. Rats were observed in their groups in two different spatial settings, and proximity and affiliative behaviours were recorded. Additionally, the hierarchy within each group was assessed to test whether preferential associations are explained by the subjects' rank. To test for temporal consistency of potential social bonds, we repeated the procedure after an interval of two days. If rats show social bonds, we predicted to find preferences to stay close to a specific group member with which they would show most affiliative behaviours. Further the interaction patterns within each group should be stable over different contexts and time points. Finally, we predicted 
bonds to be dependent on hierarchy, so that especially high-ranking individuals would be favoured bonding partners.

\section{Methods}

\section{Experimental subjects and housing conditions}

We used outbred adult female wild-type Norway rats (source: Animal Physiology Department, University of Groningen, Netherlands) weighing on average 300g, at an age of 1.5 years. Directly after weaning ( 5 weeks after birth) rats were assigned to their housing group and remained there for their lifespan. The rats were individually marked with stripes on the tail using a black permanent marker (edding3000). The ambient temperature was $20^{\circ} \mathrm{C} \pm 1^{\circ} \mathrm{C}$, with a relative humidity of $50-60 \%$ and a 12:12 h light/dark cycle, with lights on at 20:00 hours after 30 minutes of dawn and with a respective dusk period in the morning. As rats are primarily nocturnal (Norton et al. 1975) and lack red light receptors (Yokoyama and Radlwimmer 1998), all experiments were conducted under red light conditions during the dark phase of the cycle. Rats had been accustomed to all situations (hierarchy assessment and both experimental contexts) prior to the experiment.

\section{Experimental setup}

We tested for the importance of a hierarchy structure for social bonds using 21 long-term groups of 5 female rats each, resulting in 210 dyads. To clarify whether the rats form social bonds, we performed 3 behavioural tests. First, we assessed the rank order within each group. Thereafter, rats were observed with their group members in their familiar environment (context 1: "home cage"). To test for the stability of interaction patterns across different contexts, they were then observed in a large room (context 2: "open arena"), where they could more easily avoid non-preferred group members, or cluster with their potentially bonded partners. To test whether interaction patterns are stable over time, we repeated the entire procedure ( $t_{1}$ : hierarchy $\rightarrow$ home cage $\rightarrow$ open arena) after 2 days (time 
point: $t_{2}$ ). We chose a time interval of two days because female rats may change hierarchy structures (Blanchard et al. 1984; Fang and Clemens 1999) according to their 4 days oestrus cycle (Hardy 1972). The experimental timeline is depicted in Fig. 1, and the behavioural tests are described in more detail below. It was not possible to record data blind because our study involved focal observations.

\section{Hierarchy assessment}

To determine the hierarchical structure within the 21 housing groups at time points $t_{1}$ and $t_{2}$, we conducted a food-allocation test with all rats of each group (modified after Ziporyn and McClintock, 1991). This is a well-established test to determine the hierarchy structure of female rats, which may be non-linear. For this, we placed a high value food item (piece of banana; cf. Dolivo and Taborsky, 2015) covered with a perforated plastic cup in the middle of an experimental arena (80x $40 \times 40 \mathrm{~cm}$, made of glass). Two out of five rats of each group were then placed in the arena. After both rats had explored the plastic cup, we removed the rats and uncovered the food item. Directly after uncovering the food item, we again placed the two cage mates into the arena at its opposite ends, both with the same distance to the available food item. The winner was defined by gaining and monopolizing the food item. In cases of uncertainty, the test was repeated. Each rat was tested against all four littermates and the order was fully randomized beforehand using the Excel function (RANDBETWEEN). Rats had been food deprived for at most 10 hours prior to testing during the inactive phase of the rats' daily cycle (whereas water access was provided ad libitum) in order to increase the motivation level, so that both partners were motivated to gain the food reward.

\section{Measuring social interaction patterns in context 1: "home cage"}

The home cage of each group was carried to a different room and all hiding possibilities (except hay) were removed. A camera (Sony-handycam, HDR-CX550) with night vision was installed above the cage, recording the rats for 70 minutes. The rats were left alone during this time to exclude any external disturbance. After ten minutes of habituation, we started the data collection for one hour from the video record. We conducted scan samples of all behaviours every two minutes and noted down the 
nearest neighbour and all affiliative behaviours between group members, such as sitting-in-contact, allogrooming, and huddling (Table 1).

\section{Measuring social interaction patterns in context 2: "open arena"}

All five rats of a group were released together in a big arena $(4 * 4 \mathrm{~m}$ for the first batch of 9 housing groups, $2.5 * 2 \mathrm{~m}$ for the second batch of 12 housing groups) containing five shelters spread over the floor. The experimenter was sitting on an elevated tower to exclude any interactions with and disturbance of the rats. As in the home cage context, we conducted the same scan sampling of proximity and affiliative behaviours.

\section{Data analysis}

Based on proximity and affiliative behaviour data (Table 1), we calculated interaction scores (after: Silk et al. 2006) for every dyad $(n=210)$, with $i=$ donor, $j=$ receiver and $i_{(\text {total) }}=$ every given behaviour of i to group members:

$$
\text { interaction score }_{\mathrm{ij}}=\left(\frac{\text { proximity }_{\mathrm{ij}}}{\text { proximity }_{\mathrm{i}(\text { total })}} \times 100\right)+\left(\frac{\text { affiliative behaviours }_{\mathrm{ij}}}{\text { affiliative behaviours }_{\mathrm{i}(\text { total })}} \times 100\right)
$$

We divided dyad values by the overall interactions of the active individual to correct for overall activity. This led to 4 interaction scores for each rat while being in the home cage and in the open arena at time points $t_{1}$ and $t_{2}$.

All analyses were done using $\mathrm{R}$ (Version: 3.1.0.). As data on dyadic interactions cannot be independent from each other, all analyses corrected for this effect and are based on dyads as the statistical unit.

To investigate whether the hierarchy changes over time, we conducted a mixed effects null model with binomial data, using the command "glmer" with the package Ime4 (Bates et al. 2015). As the response variable, we included consistency of food monopolization (the same rat of a dyad monopolized the food item at $t_{1}$ and $t_{2}$ ). To control for multiple testing of individuals, we included "dyad" and the identity of both rats of each dyad, resulting in three random effects. 
We predicted that if rats have "closely bonded partners" not all rats would similarly interact with each other, resulting in a skewed distribution of social interactions. Such skewed (e.g. j-shaped (Silk et al. 2010)) distributions indicate that most group members interact at low levels and only a small number interact at high levels, which would point toward closely bonded partners. We therefore calculated the skewness of the data and tested whether the skewness is different from zero, which is expected under a normal distribution, using a one-sample t-test. This procedure is described in detail in Crawley (2007). For calculating this general pattern of social interactions, we used the interaction scores summed up for both contexts and both time points for all dyads, leading to a maximum value of 800 .

To analyse the association patterns for consistency over time and context, we organized the data for each housing group in matrices for contexts and time points. The matrices were asymmetric as most of the behaviours were unidirectional, such as allogrooming and nearest neighbour data (Table 1). This resulted in four asymmetrical matrices per group (for an example, see supplementary material Table S1). We used these asymmetrical matrices to correlate behavioural interactions between contexts and time points using Mantel Z tests, which controls for dependency within and between rows and columns of the same individual, following Hemelrijk (1990). Additionally, we analysed the influence of hierarchy on association patterns using the same method. We are not aware of an Rpackage to conduct this test and therefore wrote an own script based on Hemelrijk (1990) (see supplementary material for the R script). For the Mantel Z tests, $10^{\prime} 000$ permutations were conducted, using the R-package permute (Simpson et al. 2016). The tests revealed one p-value for each housing group and each comparison. Afterwards we conducted the Fisher's combined probability test $\left(\mathrm{X}_{2 \kappa}^{2} \sim-2 \sum_{i=1}^{\kappa} \ln (p)\right)$ to check for overall significance (Sokal and Rohlf 1995). 


\section{Results}

\section{Consistency of rank order}

Rats that monopolized a food item at time point $t_{1}$ were more likely to do so also at time point $t_{2}$ within the same dyad, indicating a stable hierarchy over time (GLMM: $\beta=0.682 \pm 0.16, p<0.0001)$.

\section{Social bonds}

The null expectation, if rats do not have preferred interaction partners within their group, was that roughly $25 \%$ of all interactions should have occurred with each of the 4 cohabitants in the same cage. In accordance with this prediction of a random, i.e. unbiased interaction pattern, the mean interaction score was 197.28 , which corresponds to $24.7 \%$ of the possible maximum value (800; Fig. 2). This lack of a significant skew in the distribution of interaction partners (one-sample-t-test: $\mathrm{df}=210, p=0.50$ ) suggests that bonds between specific group members were missing.

Social relations were neither stable over contexts (Fisher's combined probability tests: home cage vs. open arena tests at $t_{1}: n=21, p=0.65$; home cage vs. open arena tests at $t_{2}: n=21, p=0.99$ ) nor over time (Fisher's combined probability test: home cage $t_{1}$ vs. $t_{2}: n=21, p=0.99$; open arena $t_{1}$ vs. $t_{2}: n=21$, $p=0.97$; Fig. 3). Rank order had no influence on social relations at any of the 4 situations (Fisher's combined probability test: home cage $\left[\mathrm{t}_{1}\right]: \mathrm{n}=21, p=0.96$; home cage $\left[\mathrm{t}_{2}\right]: \mathrm{n}=21, p=0.86$; open arena $\left[\mathrm{t}_{1}\right]: \mathrm{n}=21, p=0.72$; open arena [ $\left.\mathrm{t}_{2}\right]: \mathrm{n}=21, p=0.98$; see also Fig. S1 of the supplementary material).

\section{Discussion}

Contrary to predictions derived from the hypothesis that female Norway rats exhibit social bonds among specific group members, test subjects did not interact more often with certain individuals of their group. In addition, interaction patterns between dyads were neither stable over time nor over different contexts, and they were independent of the hierarchical structure that itself remained stable over time. Therefore, our data do not indicate that female rats form social bonds. 
In rats, both sexes form dominance hierarchies. However, in contrast to male Norway rats that form stable hierarchies mainly based on body weight, females form less stable and not strictly linear hierarchies strongly influenced by their oestrus cycle (Adams and Boice 1983; Ziporyn and McClintock 1991). Our results show that food monopolisation among female rats living in long-term stable social groups was consistent over time. Despite this stability of rank order, we did not find effects of rank on individual relationships between our test subjects.

Given that rats are highly social animals living in large groups and showing considerable levels of cooperation, it may at first glance seem surprising that there is no evidence for the existence of social bonds among individual group members. Under natural conditions, however, rats might form small subgroups within colonies which could pose an alternative to close dyadic bonds. Information about natural colonies of Norway rats is scarce and there is mixed evidence for the existence of subgroups in large rat colonies. Barnett (1963, pp 83-100) described family groups to exist only until pups are weaned, but Calhoun (1979, pp 172-176) observed the occurrence of flexible subgroups in rat colonies, with individuals sometimes belonging to several such groups. If sub-structures exist within large colonies, rats might have close relationships to all members of their subgroup and perhaps behave more cooperatively towards in-group members than to individuals not belonging to the same subgroup, instead of forming dyadic bonds. The group size in our study was small and might have resembled the size of subgroups within larger rat colonies, which can contain up to 200 individuals (Telle 1966). This might explain why our test subjects did not discriminate among the members of their group.

Our study does not suggest that the seemingly altruistic cooperation exhibited by Norway rats (e.g. Rutte and Taborsky 2008; Schneeberger et al. 2012; Dolivo and Taborsky 2015a, b; Wood et al. 2016) is governed by close social bonds. This is in line with previous evidence showing that reciprocal cooperation among rats may simply reflect their discrimination based on preceding interactions, which causes them to preferably cooperate with individuals that recently have been helpful (cf. Dolivo 
et al. 2016). This may generate fitness benefits: rats have been shown to survive longer, for instance, when reciprocating affiliative behaviours (Yee et al. 2008).

A lack of stable relationships has also been described in other social animals. Pigs, for instance, have preferred association partners over short time periods, but these relationships break down over a longer time frame (Durrell et al. 2004). Female house mice have preferred partners to communally nurse with (Weidt et al. 2008), but still most of their interactions can be explained by stochastic interactions (Perony et al. 2010). In addition many group-living lemur species develop weak or no social bonds (Kappeler 1997). In primates the existence of close and consistent social bonds in general has been challenged (Henzi et al. 2009; but see Silk et al. 2010): rather than establishing long-term relationships, individuals might also react on each other's immediate behaviour (Barrett and Henzi 2001). Such short-term contingencies may engender fitness benefits in complex societies without relying on the formation of long-term social bonds, because reciprocally cooperating social partners can be subject to correlated payoffs, which reflect immediate contingencies (Taborsky et al. 2016). Especially in societies characterized by short life-spans and generation times and by the formation of flexible fission-fusion groups, short-term contingencies might have more positive fitness effects than long-term social bonds.

We conclude that, although rats show sophisticated cooperative behaviours and contrary to the hypothesis that this may reflect the existence of stable social bonds, evidence for such relationships is lacking. This is in line with previous work suggesting that rats base their decision to cooperate with a social partner on recent encounters rather than on cumulative social experiences. It is an interesting challenge for future studies to clarify which ecological or social circumstances favour either the establishment of social bonds or more immediate responses to the behaviour of group members. 


\section{Compliance with ethical standards}

In accordance with the animal welfare regulations of Switzerland (Tierschutzverordnung Schweiz $04 / 2008$ ) the rats (weight category $300-400 \mathrm{~g}$ ) were housed in enriched cages $(80 / 50 / 37.5 \mathrm{~cm})$. Enrichment included a wooden house and board, a channel, a piece of wood to nibble, a loo roll to play, digging-material (wood shavings), nest-building material (hay), and a salt block. Food (conventional rat pellets and corn mix alternating with fresh vegetables or fruits) and water were provided ad libitum according to recommendations of the Federation of Laboratory Animal Science Associations (Forbes et al. 2007). We established small groups with five individuals per cage (Sharp et al. 2003). All rats experienced a handling procedure from early age onwards, so they were well habituated to humans and not stressed while being transported to the experimental cage or by the presence of an observer. No injuries occurred in the experiments. Approval and research permission was granted by Swiss Federal Veterinary Office under license BE25/14.

\section{Author contributions}

MKS and MT conceived and designed the study and wrote the manuscript. JN, KS, AKR and MG collected the data. MKS and LE planned the statistical analyses, which were conducted by MKS. LE wrote the R-code for all Mantel tests. All authors read and commented on the final version of the manuscript.

\section{Acknowledgments}

We thank Evi Zwygart for help with animal care, Valentina Balzarini and Michelle Gygax for drawings, and Joachim G. Frommen for comments on the manuscript. This study was funded by the SNF-grant 31003A_156152 provided to MT. 


\section{Conflict of interest}

The authors declare that they have no conflict of interest.

\section{Ethical approval}

All applicable international, national, and/or institutional guidelines for the care and use of animals were followed. This article does not contain any studies with human participants performed by any of the authors.

\section{Data availability}

All data generated or analysed during this study are included in the supplementary information files. 


\section{Literature}

Adams N, Boice R (1983) A longitudinal study of dominance in an outdoor colony of domestic rats. J Comp Psychol 97:24-33

Archie EA, Moss CJ, Alberts SC (2001) Friends and relations: kinship and the nature of female elephant social relationships. In: Moss CJ, Croze H, Lee PC (eds) The Amboseli elephants: a long-term perspective on a long-lived mammal. The University of Chicago Press, Chicago, pp 238-245

Archie EA, Moss CJ, Alberts SC (2006) The ties that bind: genetic relatedness predicts the fission and fusion of social groups in wild African elephants. Proc R Soc Lond B 273:513-522

Barnett SA (1963) The rat - a study in behavior. Aldine, Chicago

Barrett L, Henzi SP (2001) Contraints on relationship formation among female primates. Behaviour 139:263-289

Bates DM, Maechler M, Bolker BM, Walker S (2015) Fitting linear mixed-effects models using Ime4. J Stat Softw 67:1-48

Beauchamp G (1998) The effect of group size on mean food intake rate in birds. Biol Rev 73:449-472

Beery AK, Routman DM, Zucker I (2009) Same-sex social behavior in meadow voles: multiple and rapid formation of attachments. Physiol Behav 97:52-57

Blanchard CD, Fukunaga-Stinson C, Takahashi LK, Flannelly KJ, Blanchard RJ (1984) Dominance and aggression in social groups of male and female rats. Behav Process 9:31-48

Braun A, Bugnyar T (2012) Social bonds and rank acquisition in raven nonbreeder aggregations. Anim Behav 84:1507-1515

Calhoun JB (1979) The ecology and sociolbiology of the Norway rat. U.S. Dept. of Health, Education, and Welfare, Public Health Service, Bethesda

Cameron EZ, Setsaas TH, Linklater WL (2009) Social bonds between unrelated females increase reproductive success in feral horses. Proc R Soc Lond B 106:13850-13853

Côté I. M, Poulinb R (1994) Parasitism and group size in social animals: a meta-analysis. Behav Ecol 6:159-165

Crawley MJ (2007) The R book. John Wiley \& Sons Ltd, Chichester

Crockett CM, Janson CH (2000) Infanticide in red howlers: female group size, group composition and a possible link to folivory. In: van Schaik $\mathrm{CP}$, Janson $\mathrm{CH}$ (eds) Infanticide by males and its implications. Cambridge University Press, Cambridge, pp 75-98

Crockford C, Wittig RM, Langergarber KE, Ziegler TE, Zuberbühler K, Deschner T (2013) Urinary oxytocin and social bonding in related and unrelated wild chimpanzees. Proc $R$ Soc $B$ 280:20122765

Davis DE (1953) The characteristics of rat populations. Q Rev Biol 28:373-401

Dolivo V, Rutte C, Taborsky M (2016) Ultimate and proximate mechanisms of reciprocal altruism in rats. Learn Behav 44:223 
Dolivo V, Taborsky M (2015a) Norway rats reciprocate help according to the quality of help they received. Biol Lett 11:20140959

Dolivo V, Taborsky M (2015b) Cooperation among Norway rats: the importance of visual cues for reciprocal cooperation, and the role of coercion. Ethology 121:1071-1080

Durrell JL, Sneddon IA, O'Connell NE, Whitehead H (2004) Do pigs form preferential associations? Appl Anim Behav Sci 89:41-52

Fang J, Clemens LG (1999) Contextual determinants of female-female mounting in laboratory rats. Anim Behav 57:545-555

Forbes D, Blom H, Kostmitsopoulus N, Moore G, Perretta G (2007) Euroguide on the accommodation and care of animals used for experimental and other scientific purposes. Federation of European Laboratory Animal Science Associations, London

Foster WA, Treherne JE (1981) Evidence for the dilution effect in the selfish herd from fish predation on a marine insect. Nature 293:466-467

Gheusi G, Goodall G, Dantzer R (1997) Individually distinctive odours represent individual conspecifics in rats. Anim Behav 53:935-944

Gilbert C, Robertson G, Le Maho Y, Naito Y, Ance A (2006) Huddling behavior in emperor penguins: dynamics of huddling. Physiol Behav 88:479-488

Hardy DF (1972) Sexual behavior in continuously cycling rats. Behaviour 41:288-297

Hemelrijk CK (1990) Models of, and tests for, reciprocity, unidirectionality and other social interaction patterns at a group level. Anim Behav 39:1013-1029

Henzi SP, Lusseau D, Weingrill T, van Schaik CP, Barrett L (2009) Cyclicity in the structure of female baboon social networks. Behav Ecol Sociobiol 63:1015-1021

Herskin J, Steffensen JF (1998) Energy savings in sea bass swimming in a school: measurements of tail beat frequency and oxygen consumption at different swimming speeds. J Fish Biol 53:366-376

Hinde RA (1976) Interactions, relationships and social structure. Man 11:1-17

Holekamp KE, Cooper SM, Katona Cl, Berry NA, Frank LG, Smale L (1997) Patterns of association among female spotted hyenas (Crocuta crocuta). J Mammal 78:55-64

Hurst JL, Barnard CJ, Nevison CM, West CD (1997) Housing and welfare in laboratory rats: welfare implications of isolation and social contact among caged males. Anim Welfare 6:329-347

Hurst JL, Barnard CJ, Nevison CM, West CD (1998) Housing and welfare in laboratory rats: the welfare implications of social isolation and social contact among females. Anim Welfare 7:121-136

Janson CH (1988) Food competition in brown capuchin monkeys (Cebus apella): quantitative effects of group size and tree productivity. Behaviour 105:53-76

Kappeler PM (1997) Determinants of primate social organization: comparative evidence and new insights from Malagasy lemurs. Biol Rev 72:111-151

Koenig WD, Dickinson JL (2016) Cooperative breeding in vertebrates: studies of ecology, evolution, and behavior. Cambridge University Press, Cambridge 
Krafft B, Colin C, Peignot P (1994) Diving-for-food: a new model to assess social roles in a group of laboratory rats. Ethology $96: 11-23$

Lusseau D, Schneider K, Boisseau OJ, Haase P, Slooten E, Dawson SM (2003) The bottlenose dolphin community of doubtful sound features a large proportion of long-lasting associations: can geographic isolation explain this unique trait? Behav Ecol Sociobiol 54:396-405

Massen JJM, Sterck EHM, de Vos H (2010) Close social associations in animals and humans: functions and mechanisms of friendship. Behaviour 147:1379-1412

Melis AP, Hare B, Tomasello M (2006a) Chimpanzees recruit the best collaborators. Science 311:12971300

Melis AP, Hare B, Tomasello M (2006b) Engineering cooperation in chimpanzees: tolerance constraints on cooperation. Anim Behav 72:275-286

Norton S, Culver B, Mullenix P (1975) Development of nocturnal behavior in albino rats. Behav Biol 15:317-331

Perony N, König B, Schweitzer F (2010) A stochastic model of social interaction in wild house mice. In: Proceedings of the European Conference on Complex Systems 2010, https://www.sg.ethz.ch/media/publication_files/paper_eccs.pdf

Rutte C, Taborsky M (2008) The influence of social experience on cooperative behaviour of rats (Rattus norvegicus): direct vs generalised reciprocity. Behav Ecol Sociobiol 62:499-505

Schino G (2001) Grooming, competition and social rank among female primates: a meta-analysis. Anim Behav 62:265-271

Schino G, Aureli $F$ (2016) Reciprocity in group-living animals: partner control versus partner choice. Biol Rev 1-8

Schneeberger K, Dietz M, Taborsky M (2012) Reciprocal cooperation between unrelated rats depends on cost to donor and benefit to recipient. BMC Evol Biol 12:41

Schuster R, Perelberg A (2004) Why cooperate? An economic perspective is not enough. Behav Process 66:261-277

Schweinfurth MK, Stieger B, Taborsky M (accepted) Experimental evidence for reciprocity in allogrooming among wild-type Norway rats. Sci Rep

Seyfarth RM, Cheney DL (2012) The evolutionary origins of friendship. Annu Rev Psychol 63:153-177

Sharp J, Zammit T, Azar T, Lawson D (2003) Stress-like responses to common procedures in individually and group-housed female rats. J Am Assoc Lab Anim Sci 42:9-18

Silk JB (2002) Using the "f"-word in primatology. Behaviour 139:421-446

Silk JB, Altmann J, Alberts SC (2006) Social relationships among adult female baboons (Papio cynocephalus) I. Variation in the strength of social bonds. Behav Ecol Sociobiol 61:183-195

Silk JB, Beehner JC, Bergman TJ, Crockford C, Engh AL, Moscovice LR, Wittig RM, Seyfarth RM, Cheney DL (2010) Female chacma baboons form strong, equitable, and enduring social bonds. Behav Ecol Sociobiol 64:1733-1747 
Silk JB, Beehner JC, Bergman TJ, Crockford C, Engh AL, Moscovice LR, Wittig RM, Seyfarth RM, Cheney DL (2009) The benefits of social capital: close social bonds among female baboons enhance offspring survival. Proc R Soc Lond B 276:3099-3104

Simpson GL, Bates DM, Oksanen J (2016) permute: Functions for generating restricted permutations of data. Available at https://cran.r-project.org/web/packages/permute/index.html

Sokal RR, Rohlf FJ (1995) Biometry, 3rd edn. W. H. Freeman \& Company, New York

Taborsky M, Frommen JG, Riehl C (2016) Correlated pay-offs are key to cooperation. Philos T Roy Soc B 371:20150084

Tan L, Hackenberg TD (2016) Functional analysis of mutual behavior in laboratory rats. J Comp Psychol 130:1-11

Telle H (1966) Beitrag zur Erkenntnis der Verhaltensweise von Ratten, vergleichend dargestellt bei Rattus norvegicus und Rattus rattus. Z Angew Zool 53:129-196

Val-Laillet D, Guesdon V, von Keyserlingk MAG, de Passillé AM, Rushen J (2009) Allogrooming in cattle: relationships between social preferences, feeding displacements and social dominance. Appl Anim Behav Sci 116:141-149

Wagner RH (1992) The pursuit of extra-pair copulations by monogamous female razorbills: how do females benefit? Behav Ecol Sociobiol 29:455-464

Weidt A, Hofmann SE, König B (2008) Not only mate choice matters: fitness consequences of social partner choice in female house mice. Anim Behav 75:801-808

Weingrill T (2000) Infanticide and the value of male-female relationships in mountain chacma baboons. Behaviour 137:337-359

Wood RI, Kim JY, Li GR (2016) Cooperation in rats playing the iterated Prisoner's dilemma game. Anim Behav 114:27-35

Yee JR, Cavigelli SA, Delgado B, McClintock MK (2008) Reciprocal affiliation among adolescent rats during a mild group stressor predicts mammary tumors and lifespan. Psychosom Med 70:10501059

Yokoyama S, Radlwimmer FB (1998) The "five-sites" rule and the evolution of red and green color vision in mammals. Mol Biol Evol 15:560-567

Ziporyn T, McClintock MK (1991) Passing as an indicator of social dominance among female wild and domestic Norway rats. Behaviour 118:26-41 


\section{hierarchy test}

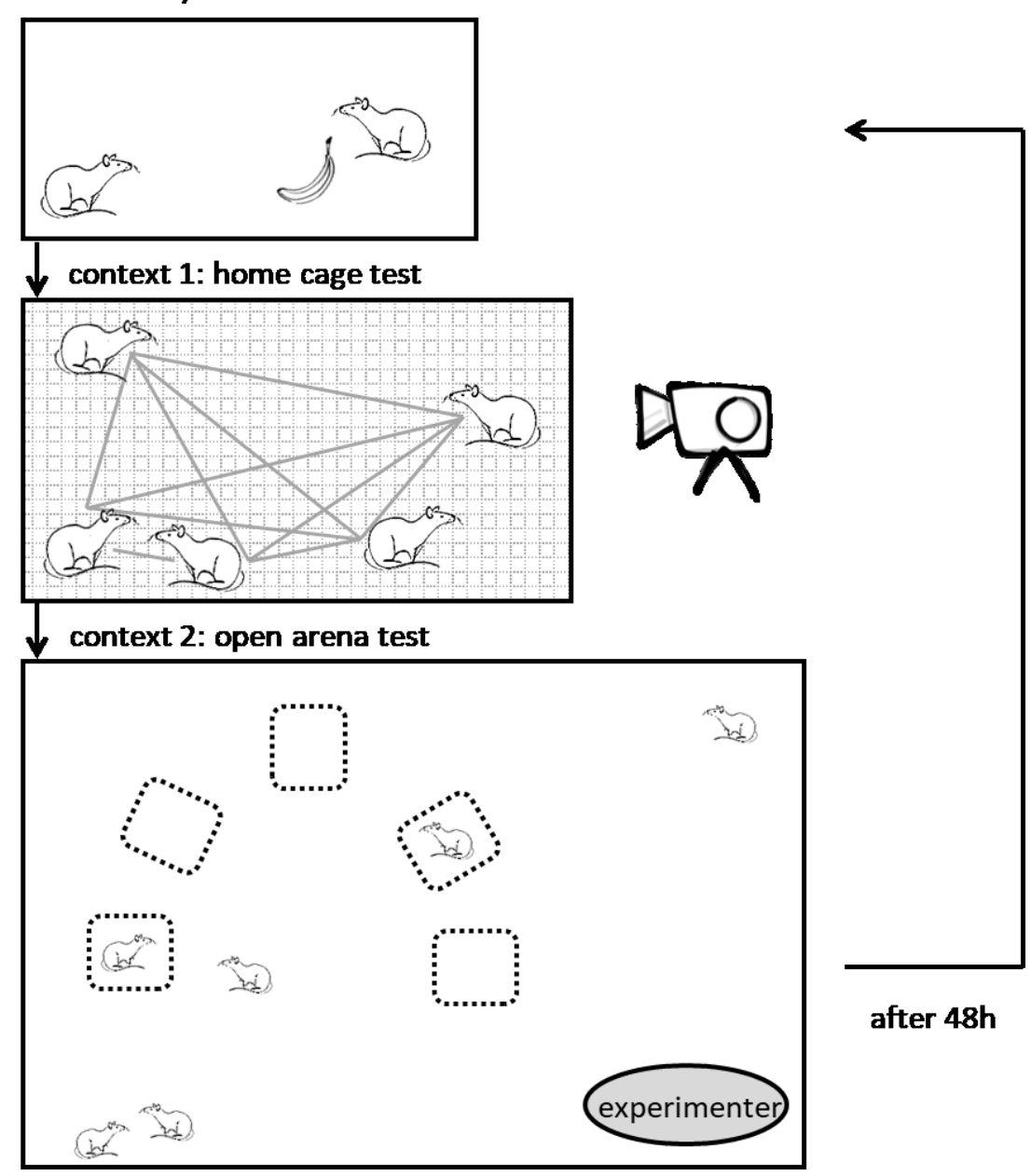

\section{Fig.1 Experimental setup}

First, the hierarchical structure of each housing group $(n=21)$ was assessed by a food monopolization test. The individual monopolizing the food item (here a piece of banana) was assumed to be dominant over the partner. Thereafter, rats were videotaped for 1 hour in their home cage. Here, we recorded which individual interacted with whom and which behaviours were exchanged. Finally, rats were released in an open arena with 5 huts as shelters for hiding (indicated by dashed rectangles). Here, the experimenter directly observed interactions between rats of the same housing group. After 48 hours, the whole procedure was repeated. All possible dyads of 21 housing groups involving 5 individuals each were tested 


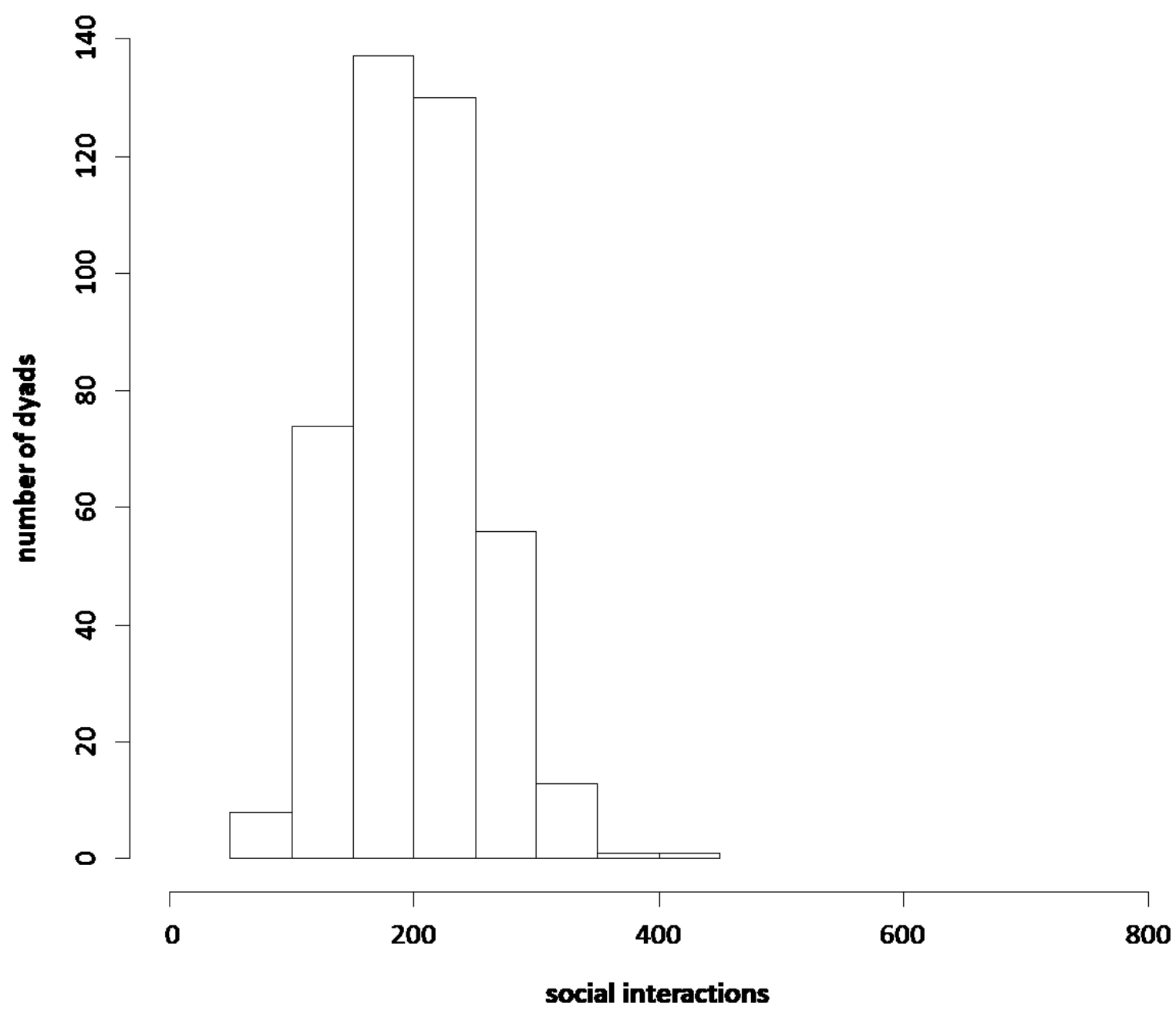

Fig.2 Distribution of social interaction frequencies within groups of 5 rats

Summing up social interaction scores of each rat from two social contexts at two points in time generated 420 data points resulting from 210 dyads (passive and active partners as some behaviours are unidirectional such as allogrooming). A value of 800 on the abscissa would indicate that two rats had exclusively interacted with each other, while a value of 0 would indicate no interactions at all. The mean value of social interactions between two randomly drawn partners of a group was 197.28 , with a maximum of 421 . Hence, the interaction frequencies between group members were not significantly skewed, indicating that all group members interacted at similar levels with each other. 

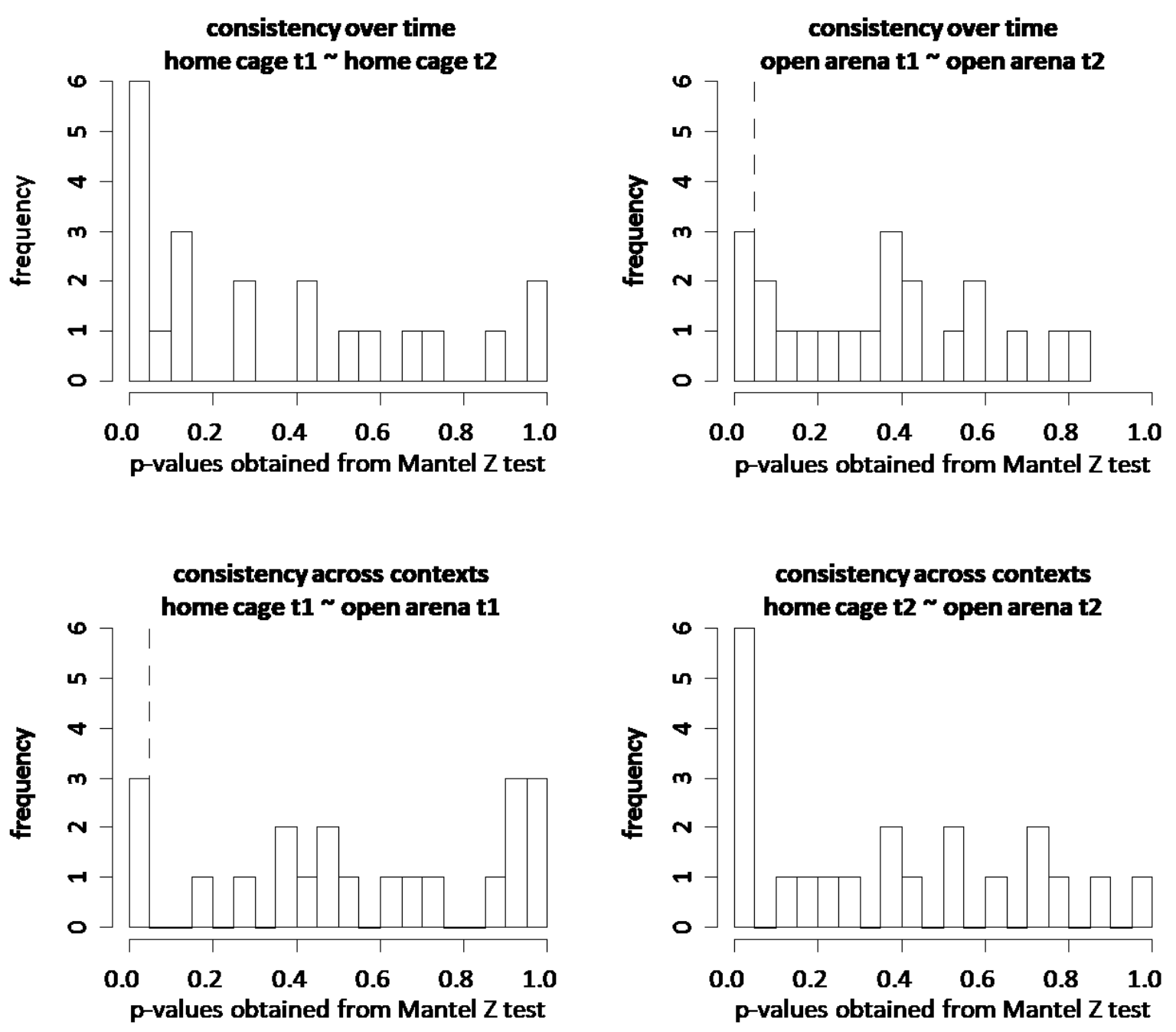

Fig.3 Consistency of social relations over time and in different contexts

$P$-values obtained from Mantel $Z$ tests for each comparison, with the respective $\alpha$-levels indicated by dotted lines. Matrices of each housing group $(n=21)$ were compared within two contexts (home cage \& open arena) over time to test for temporal consistency (upper graphs). Furthermore, matrices were compared over the two different contexts at time points $t_{1}$ and $t_{2}$ to test for consistency over different contexts (lower graphs). 


\section{Table 1 Ethogram of behavioural data for interaction scores}

Rats were observed while interacting with their group members in two contexts. They were observed while being in their home cage and in a large open arena. In both situations, we noted down who was the nearest neighbour of whom and which affiliative behaviours occurred (sitting-incontact, allogrooming, huddling)

\section{proximity}

\begin{tabular}{l|l} 
nearest & Nearest neighbours are rats that are
\end{tabular} neighbour closest to each other but not further away than $20 \mathrm{~cm}$, which is approximately the head-body length of rats. Note that nearest neighbours are not necessarily the same for both individuals ( $B$ is the nearest neighbour for $A$, but $C$ is the nearest neighbour for B).

\section{affiliative behaviours}

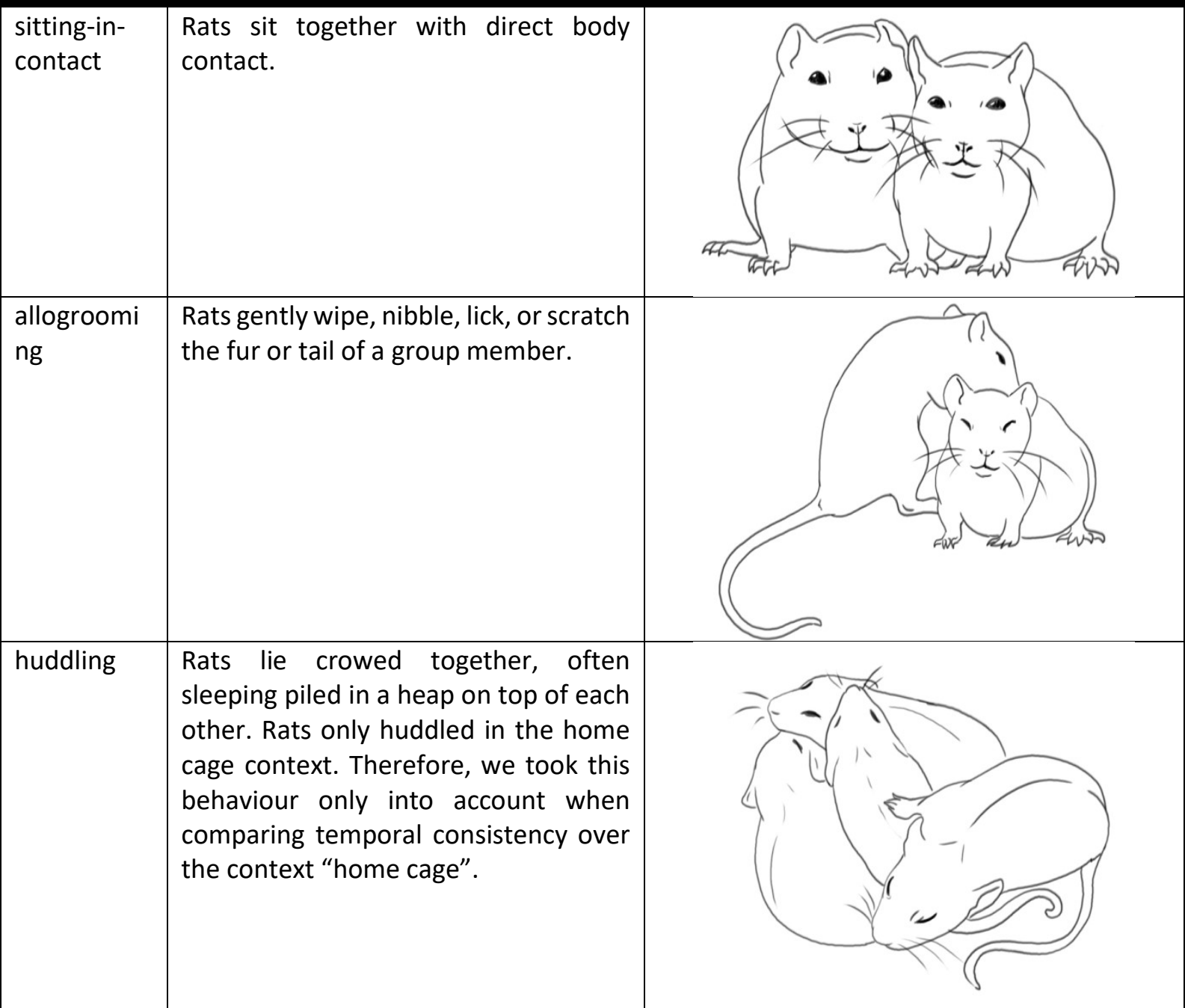




\section{R-script for Mantel test}

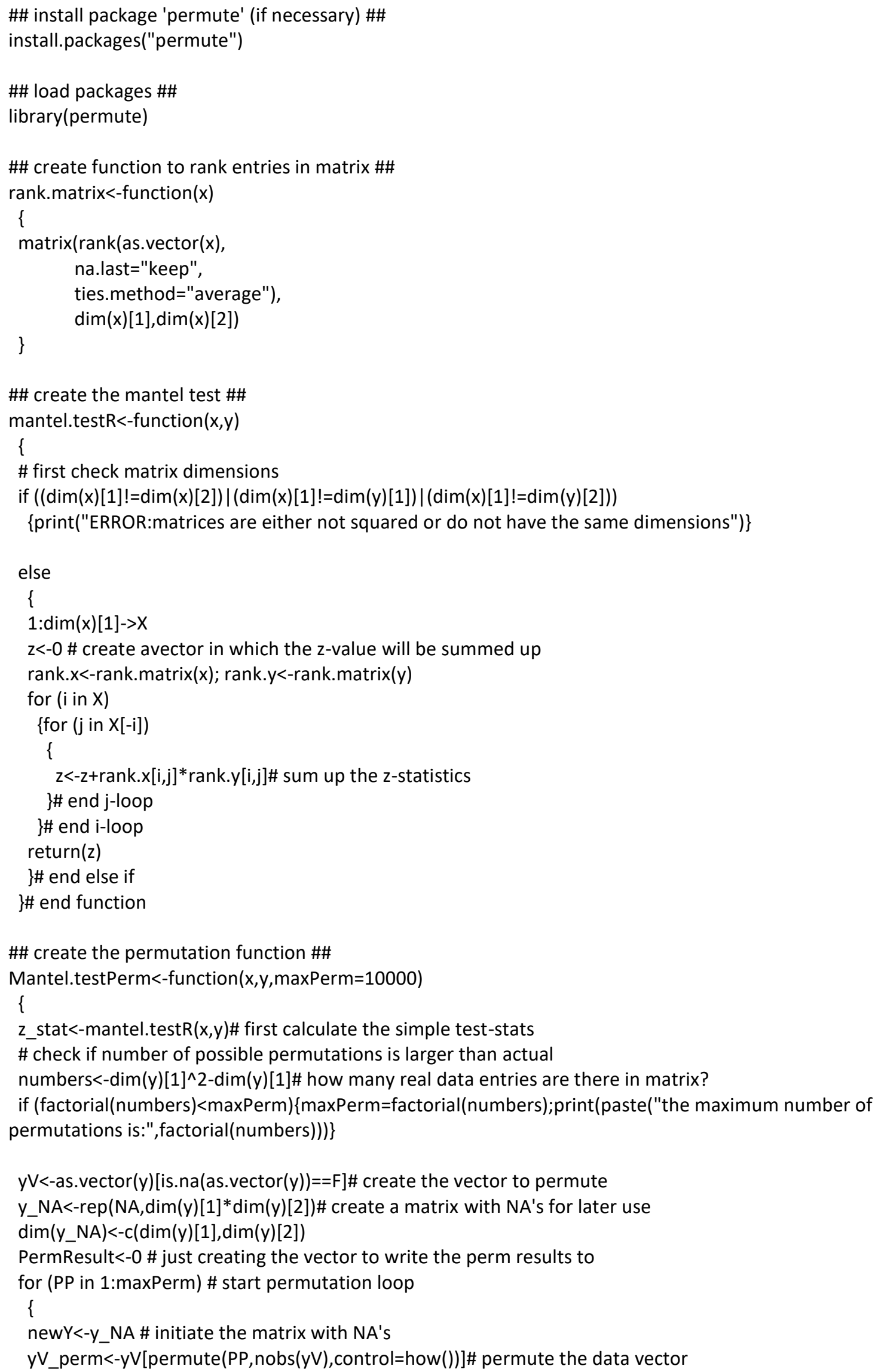




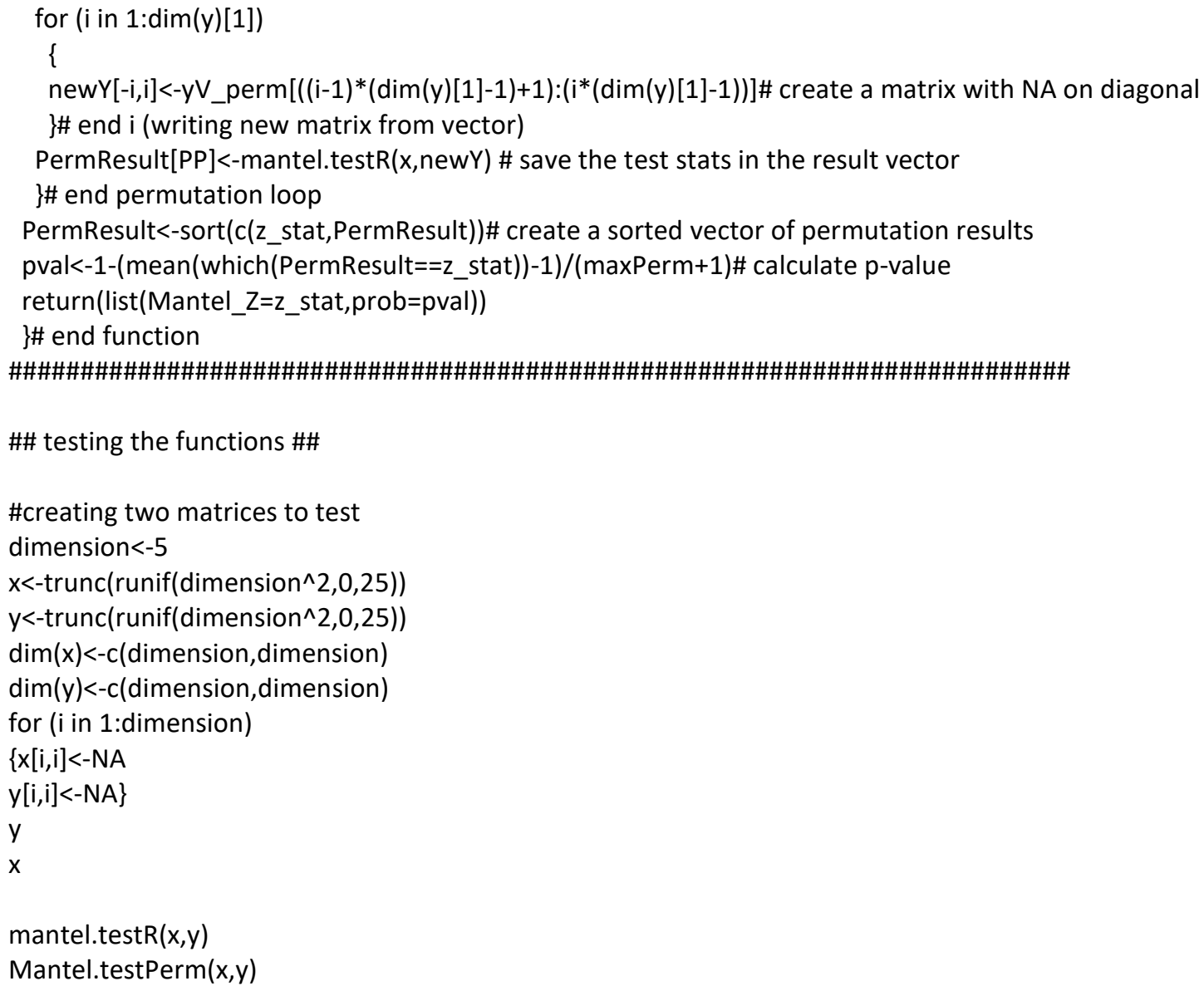


influence of rank on social bond (home cage[t1]) influence of rank on social bond (open arena[t1])

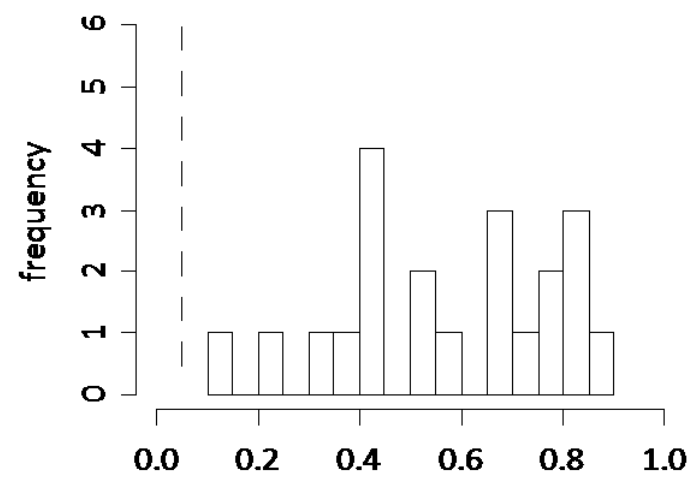

p-values obtained from Mantel Z test

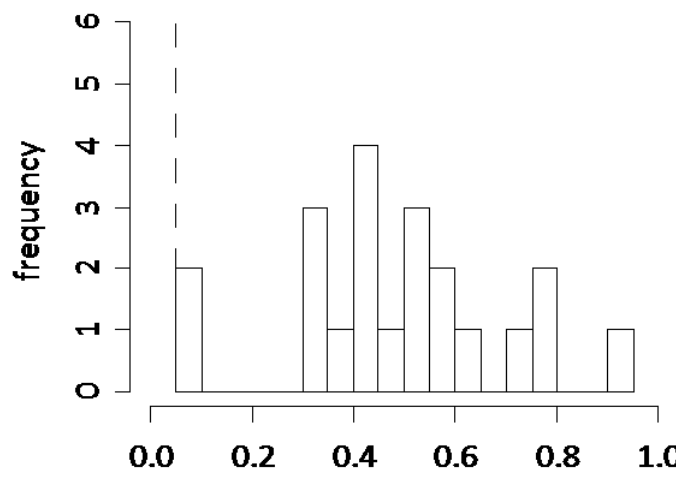

p-values obtained from Mantel Z test

influence of rank on social bond (home cage[t2]) influence of rank on social bond (open arena[t2])

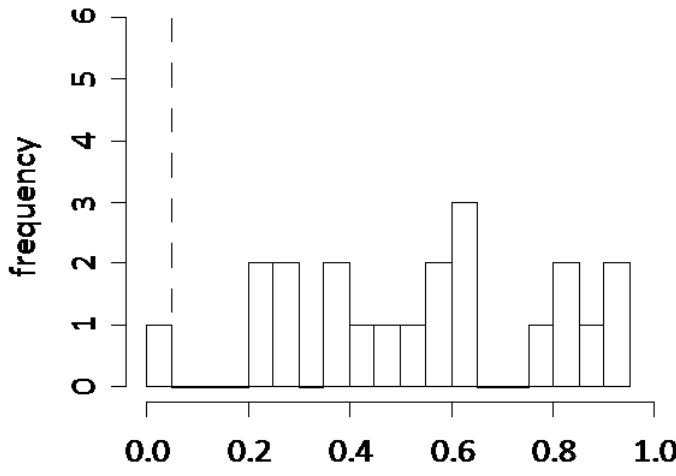

p-values obtained from Mantel Z test

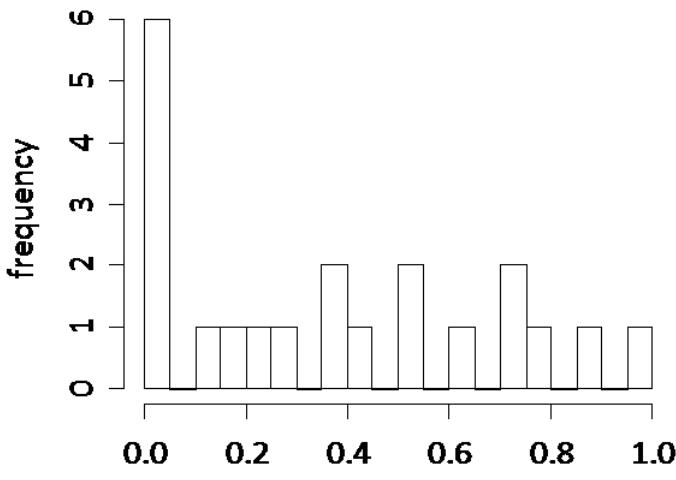

p-values obtained from Mantel Z test

Fig. S1 Influence of rank on social relationship in dependence of context and point in time

The rank of cage mates influenced their social relationship index neither in in the home cage at time point $t_{1}$ nor at $t_{2}$. The same was true for the second context in the open arena. 


\section{Table S1 Example of a social bond indices matrix}

All groups consisted of 5 individuals. In the example they are referred to as $C 1, C 2, C 3, C 4$ and $C 5$. Every dyad has two values, as members of the dyad have active and passive values. For instance, C5 has a bond index score of 93.4 with $\mathrm{C} 1$, whereas the active score of $\mathrm{C} 1$ with $\mathrm{C} 5$ is lower (68.1).

Different values are possible as some behaviours are unidirectional, for instance C5 might have groomed $\mathrm{C} 1$, which remained unreciprocated by $\mathrm{C} 1$. Asymmetrical matrices are characterized by different values above and below the "NA line". Because matrices were asymmetrical, we used the respective Mantel $Z$ test that permutated all values and not one half as it would be appropriate in symmetrical matrices.

\begin{tabular}{llllll} 
& C1 & C2 & C3 & C4 & C5 \\
C1 & NA & 72.72727 & 66.07143 & 67.27273 & 93.39339 \\
C2 & 30.55028 & NA & 17.14286 & 42.72727 & 13.51351 \\
C3 & 46.67932 & 27.27273 & NA & 47.27273 & 46.24625 \\
C4 & 54.64896 & 72.72727 & 67.14286 & NA & 46.84685 \\
C5 & 68.12144 & 27.27273 & 49.64286 & 42.72727 & NA \\
\hline
\end{tabular}

